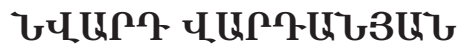

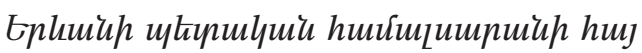

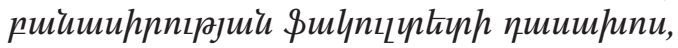

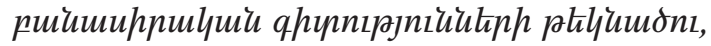

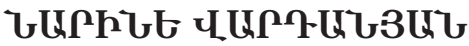

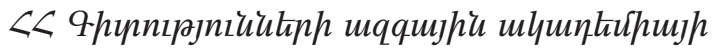

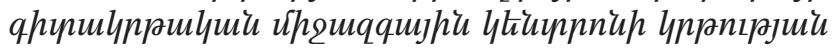

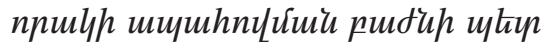

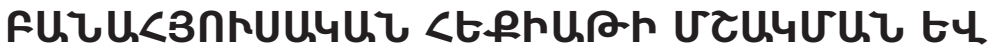

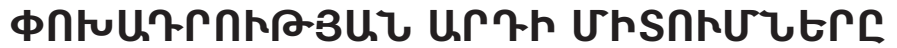

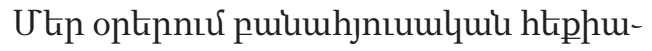

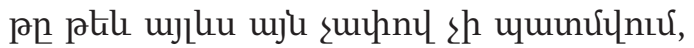

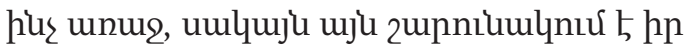

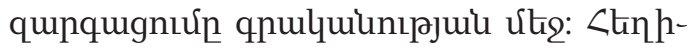

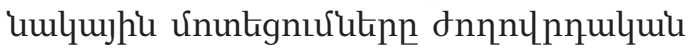
ujnıphi ujuop purulquil unupptip tiù

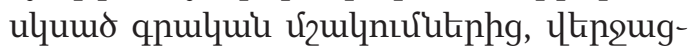

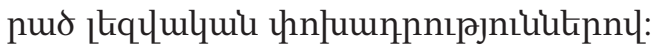

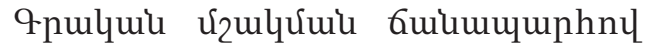

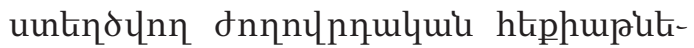

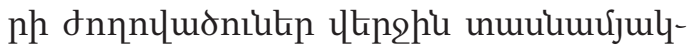

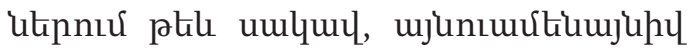

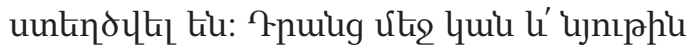

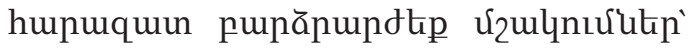
jnıpuinhu htinhumlqujhi ununtgnıúlutnnц (U. Pn乞wupjuiu, L. Jutįnjuiu), l' pnцnnnlhi unn no upunuunцnn ulqpnilputinnl

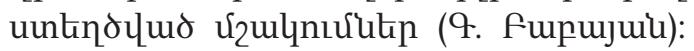
npn2 qnnnitip bl ltipupưuunuunnnuर

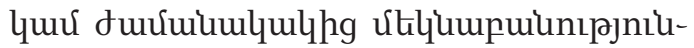

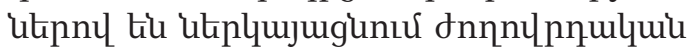
hujuih htppupitiph ujnıdtiknp (U.Mu-

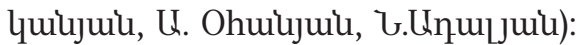

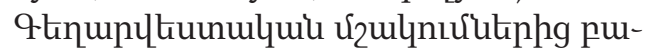

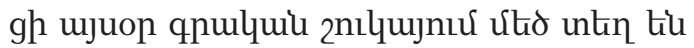

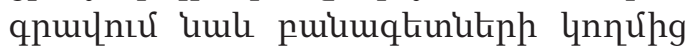

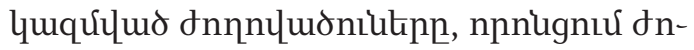

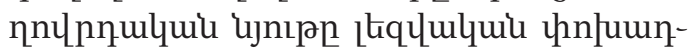

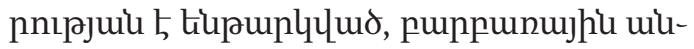

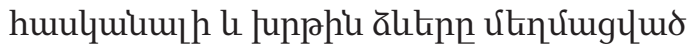
tiu (U. Guqhujuul, ৮. Lulnnjuiu, U. உu- qhjuiu, U. Ч.upquiujuiu): Ujuuphuh huinnu-

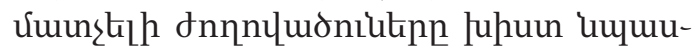

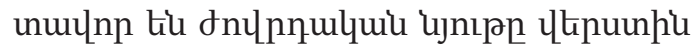

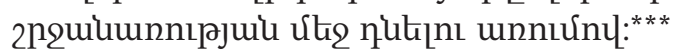

cuilqnıgujhi puntip h upunuhwj-

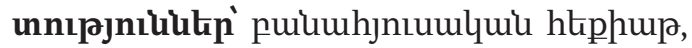
qnulyui htphwas, onnnцnпulquiu htiphupltip, htppupp jtiqunбulqui unumăuwhuunlnıpjnilutip

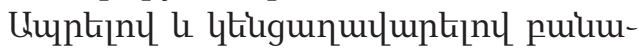

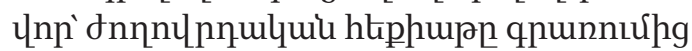
htinn luu sh numunnuर hp jnıpultipu qn-

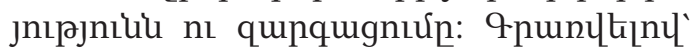

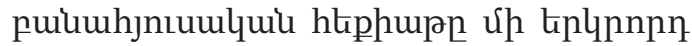

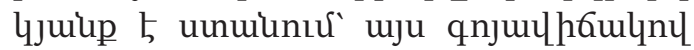
lu zupnituwlitind ătinp ptipta unpuiunp unupptinulutip` htphuph htinhumlüjh

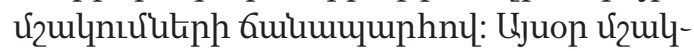
luo htiphuplitip uukinl huuluuinuর tiup npulquulia puцulquil unupptip uшunnứukp. l' pupănupdtip qnulquiu-qk-

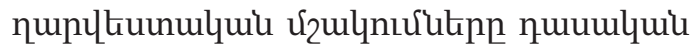

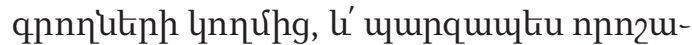

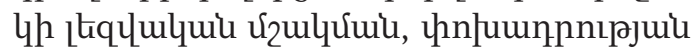

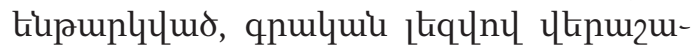

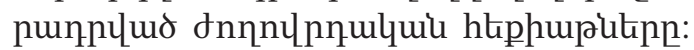

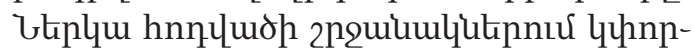
atiup punhwiunıp lknund nıpluqdil qunqugúuiu uju hhứumlumi uhunnứut-

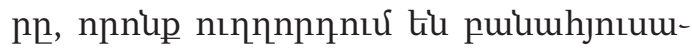

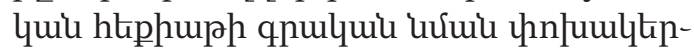




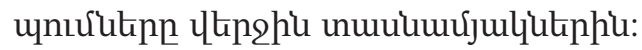

fuiumhnnunıрjuil qunqugưuiu ukn-

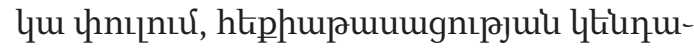

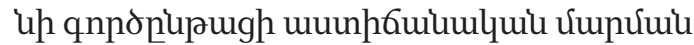

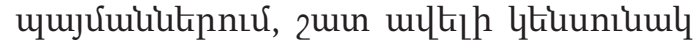

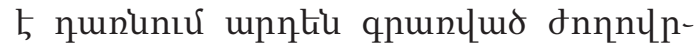
nulumu htphupn: «<uj dnnnцnпulquiu

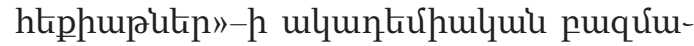
huunnpjulynuर gnuju untuum htiphupitinn

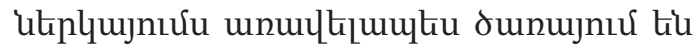

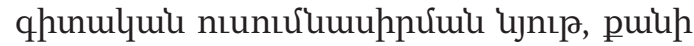

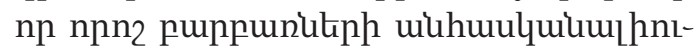

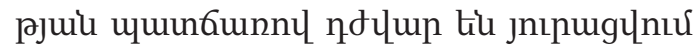

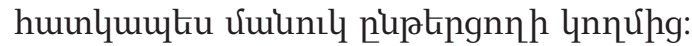
Unue 5 quilhu unntel qnunluo hulu-

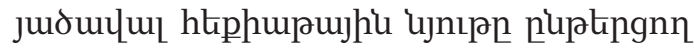

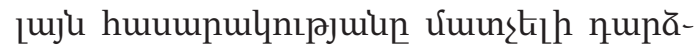

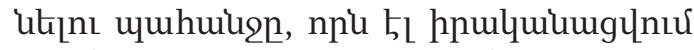

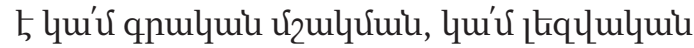

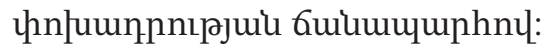

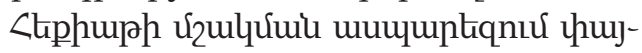
nilu ătinpptipnứutip niukgurd huj qnnn-

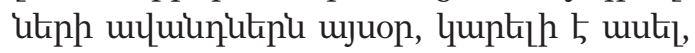

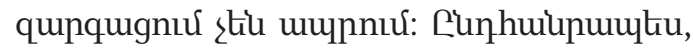

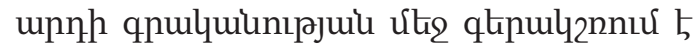
hinhumulujhu htphupn, hul onnnцnпu-

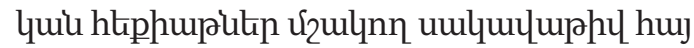

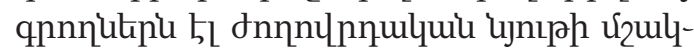

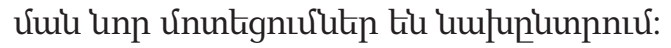

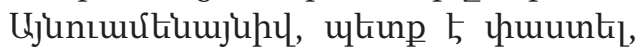
np ujuon unumqnцnn qtinunцthunulumi

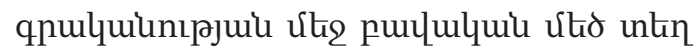
tiu qpulnnu hting htphwplitph puquum-

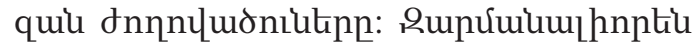
ukplqu hpulquinıpjuiu utig, tipp puptin-

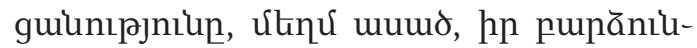

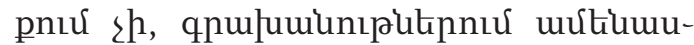

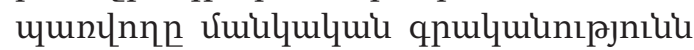

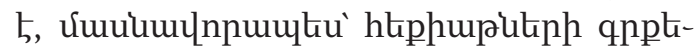
nn: Uju huiuquưuuipn nqunpnıu 5 unupptip hpuunupulısnıpjniuktiph` qupl unul

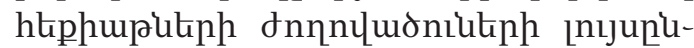

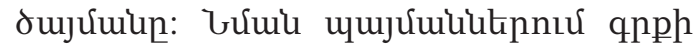
2nılünıu hujunulnıर tiu htiphupitiph

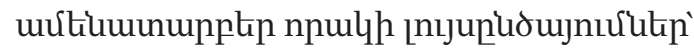

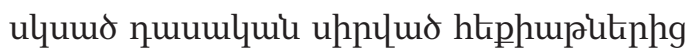

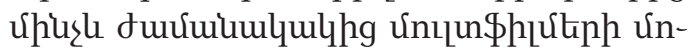

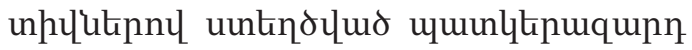
qnpnujlutipu ne qnilumqupnúuiu untinnt-

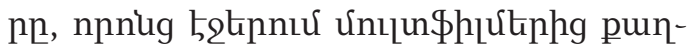

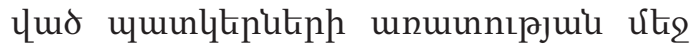

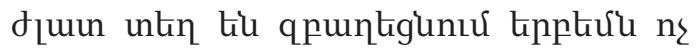
ujupuil 6urulnnl b qnuqkin zupunn-

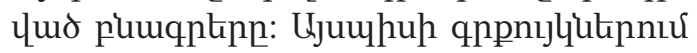

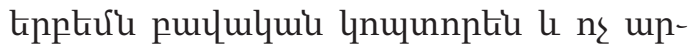

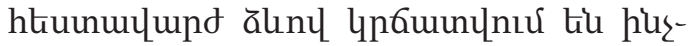
uktu huj, ujhumku bl mpnumuhưulumul nuuulquiutinh hujunup htphuplitinn,

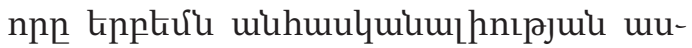

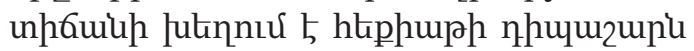
nı pnцuinumunıpjniun:

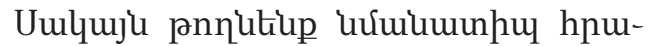
unupulnıрjnılukten, npnig puinıpjniuu ujdu vitp uuwumulhg nnıpu 5 , le ulte-

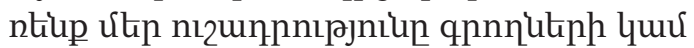

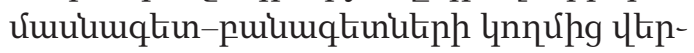

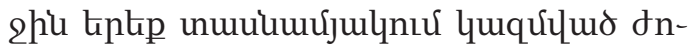

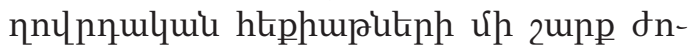

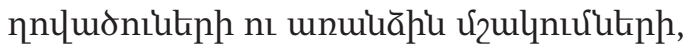

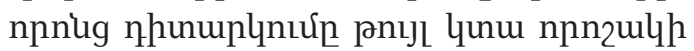

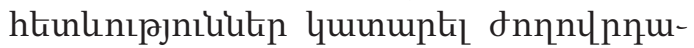

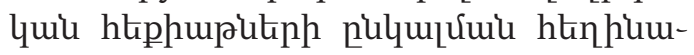
qujhu únuntgnứutiph цtipuptipjur:

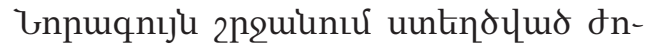

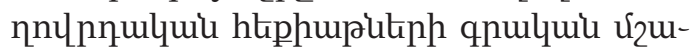
lnứukiph dnnnцuonituknhg ujuop, huun-

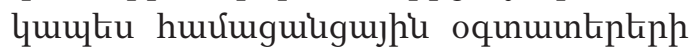
2nquiunuर, uर्utihg upnum lu puptinglnn

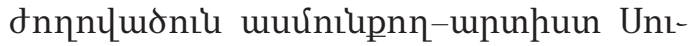

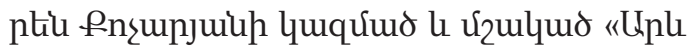
hŁphup»" (1979) qhppu 5: Ujuop hujluuuui

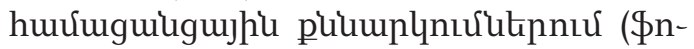

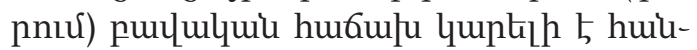

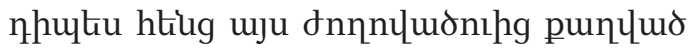
১nnnupnulumu htppumbltiph pumqptiph:

tpte qnulquinıpjuiu utis htphupditip

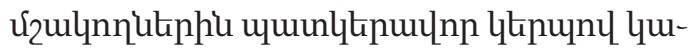

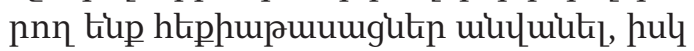

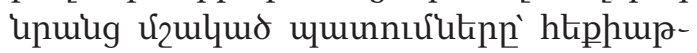
utpp unp «numptipuluthp», шuщu U.Pn- 


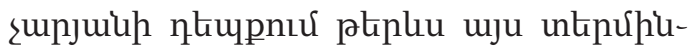

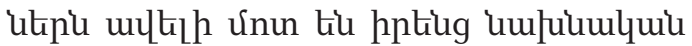
hơuunuulnpर्umin, puith np htppuptitep

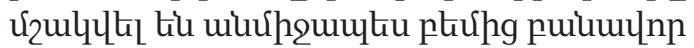

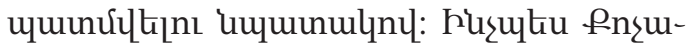

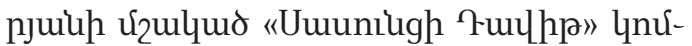

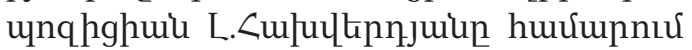
5 "Uuuuu dntpp» ú unp unupptipuly

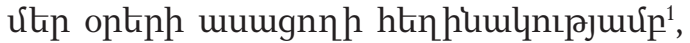

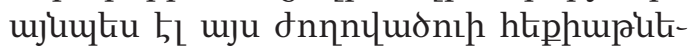

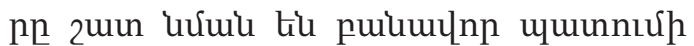
unp unupptipulutiph: onnnlpnulun-

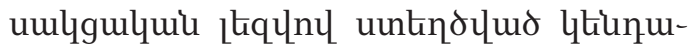

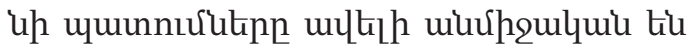

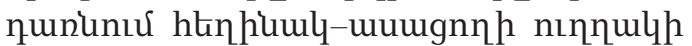

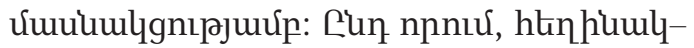

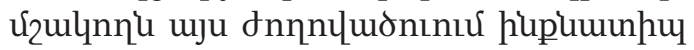
ulqpniup 5 lhpunntu. 'uu htppuplet-

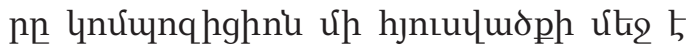

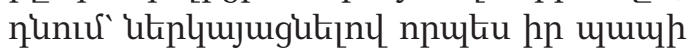

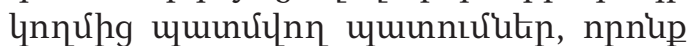

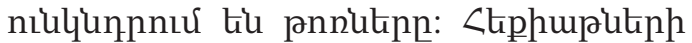

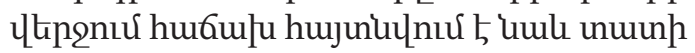

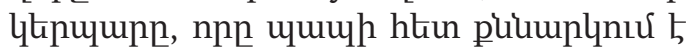
htppurp quinuщump:

Tuшu hkphupp np purưưurgntig, uutig.

- Stuuip, nulkphrn nug lukinpugntig bu puquunphi:

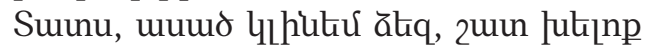
luhl 5 :

- Ulunn,- - uukg, - puquunnp jukןnpuiuuln nipu w: 2tu hơuluni̊u, np puquunph রhpnıpp uшjuunulyuiukph nı hu-

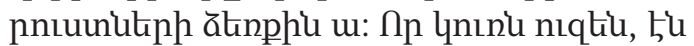
lnınp 2nın lunuil:

- nıptứu htiphupd bl suutíu,- utпuguц щщшщи:

- 25,- - uukg unuunu,- hish \&5, hkphuрр пuu u junnutphi, uर्um puquцnph lukinpuiuuln thitinn puiu sh:

- Pns $5 l$ thu upnup, uj luhl, - uukg щищии:

${ }^{1}$ L. Culuultipnjuil, 4nưunqhghuil Unıptiu ensu-

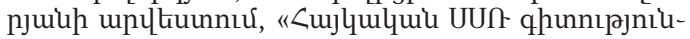

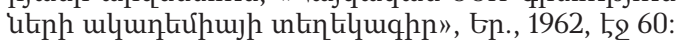

hhupltì unпun w:

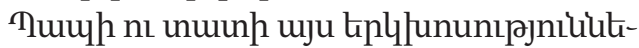
nn niulyunphi luư puptingnnhu oqunıu tiu

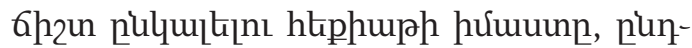

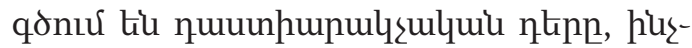
uka tumb uinınnulyhnptít umpumumun-

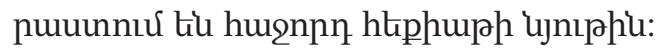

onnnцnпulqui htphupitinh úulnứutipn úh ứpnngulqui lnưurnqhghni 2nquilh utis hjnıutint le npuitg «uw-

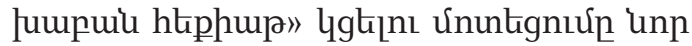
25. ujuuhup npulinpnư untiuntu tiup ntin unupuluuu «<uque no úp qh2tinutnnเư⿱乛龰:

ftứng ukplumugutuns ulqpniuph

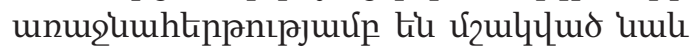
puunkpuqtion-npuưuunnınq fousuunnıp

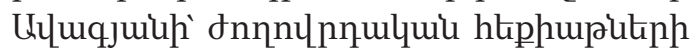
ujnıdtukph hhưpny uuntindumo uhtuut-

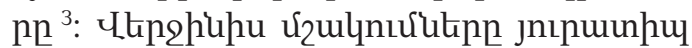
tiu upuunl, np htnhumln v2ulyuuu 5

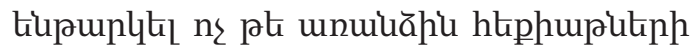
ujnıdtikten, wj ú uphtiup 2nquiumlnnu hųnnnupun únuhjnıutel 5 ú puiuh htphup, npnup qunuщumulquiu uúpnn-

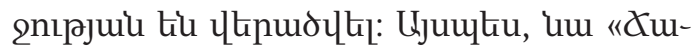

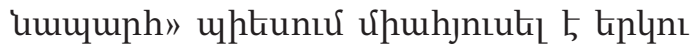

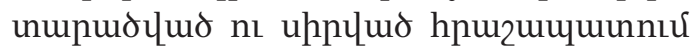
htiphuphtip` Opums lunukinh le unu uгu-

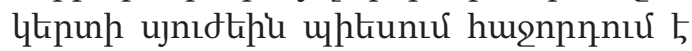

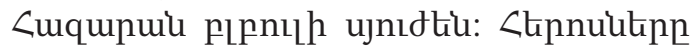

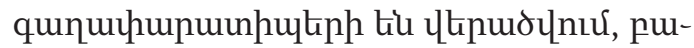
nnı, quntighlh no puenıрjuiu qnupp tinptứu htnhumbhi nınпulh junhtiph nı

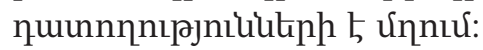

Unuultl puquiuquil, pujg nupajul qunuщumupuinntiu hpup úpuhjnıuцuo ujnıdtiutinnl 5 qunquiunıu

\footnotetext{
2 Abd-El-Hameed Hawas, A prologue Tale as Manifesto Tale: Establishing a Narrative Literary Form and the Formation of Arabian Nights, 'Marvels \& Tales, vol. 21, No. 1, Fary Tales, Printed Texts and Oral Tellings, 2007, pp. 65-77.

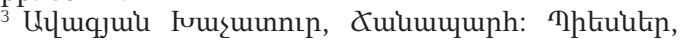

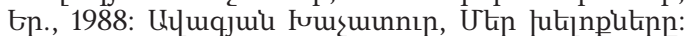

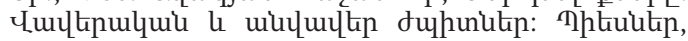
ثp., 2002:
} 


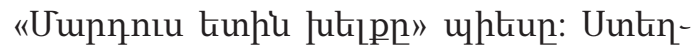

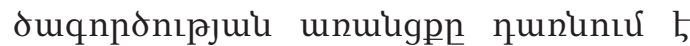
hpuuquinnuर hłphupp hujunih htpnup' bukjugh, Gupupl, humpuqtiun, nph znıne hjnıuцud unupptip ujnıdtitipn htinhuu-

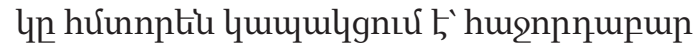

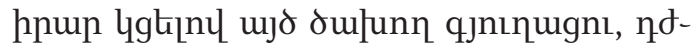

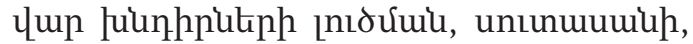
puquцnph quर्umlnn unglum, qunh, pjnıuujh nı lnıqh htphupujhl ujnıdtits-

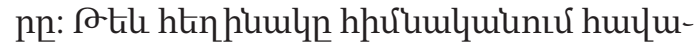

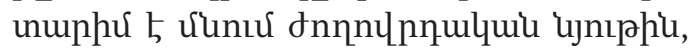

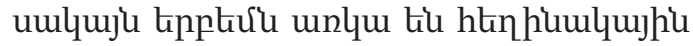

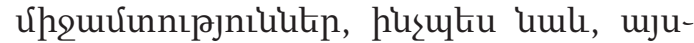
unkn lu tipptứu wnqu pupnqn le pupnjüununıрjnilu:

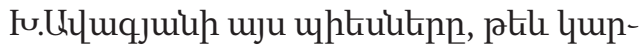
btu pte ujuop piptingnn juju huumpu-

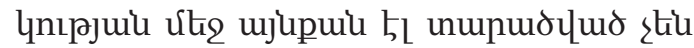
$\mathrm{l}$, guцnp, nuin upduiuцnıju quwhuunцwo

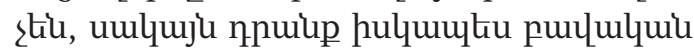
nı2uqpuul npulenpnưutup tiu huj htphu-

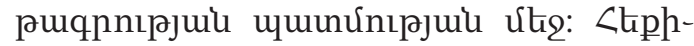
uplutpp piunpnipjniup le npuiug hưntun

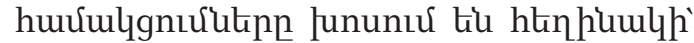
dnnnlpnuluiu htppuptitph puruint-

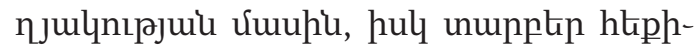

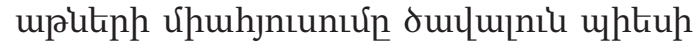

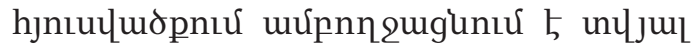
hłpnup unhup le thnцhu ukplujuguntu 5 dnnnцpпuluiu htphupp hupnıun w2luwphn:

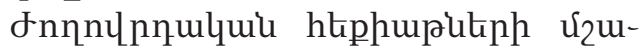

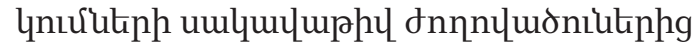

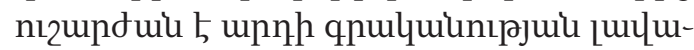

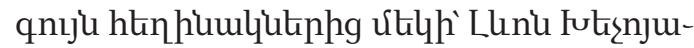
up' "Suil uquhuumulu hptrznulpn" (2002)

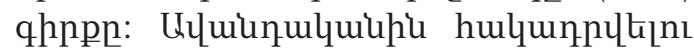

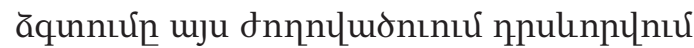

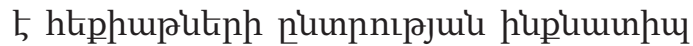
ulqpniupnu: <tinhumln htiphuputiph

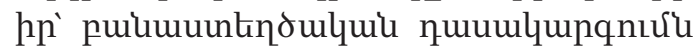

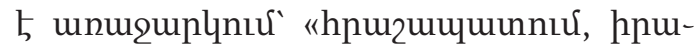

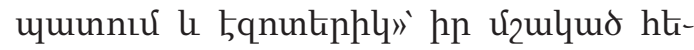
phuplutinn hưưptinnl tipnnpn luर्uph ưunnıরutup: "Funāp <ujph jtunutuphg hquo, umpnıp ns «uuunluow2usjuiu 62-

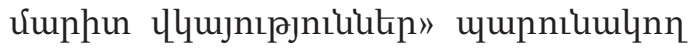

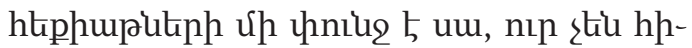

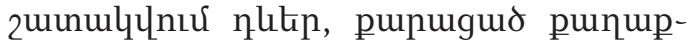

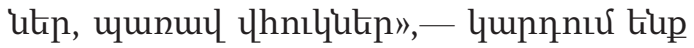

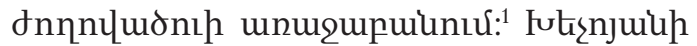

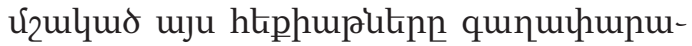

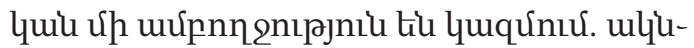
hujun 5, np htnhiuulp hp ungl sh nptil ujuuhup juinhpitup, hisuhupp til' on-

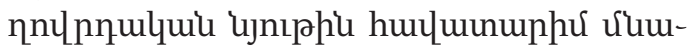
ın, puppunujhì jkqnil, dnnnlpnulpuil

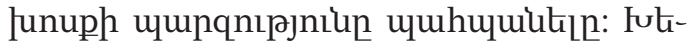
¿njuiun lnuju u2luwph 5 huiuntu dnnnuln-

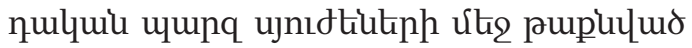

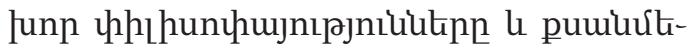
lkpnnn nuph qnnnh lunuptipnl huskg'unıর 5 npuiup' 4juiuph hơuuun, 6h2u

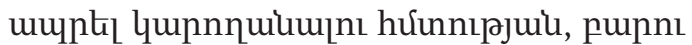
nı ¿wiph hupuptenulgnıpjuiu, pupnì npuku pugupăuly upttpp qtenulyujnıpjuil

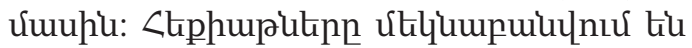

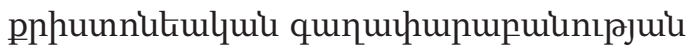
untumulyniuhg, hłnhumln hufulu ưu-

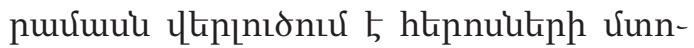

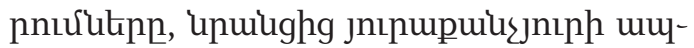

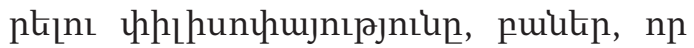
stüp quinnn quitul dnnnlpnulyuil htphupitiph puuqpkpnıর: Tuunuhulqui

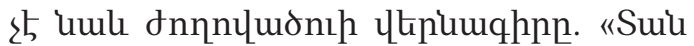

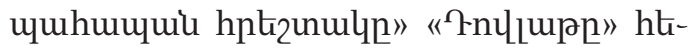
phup unpuwnplus unupptipulu 5, nрp punıpuqpulquil 5 qpp piuphuiunıp

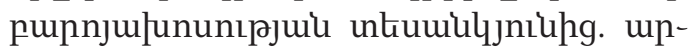

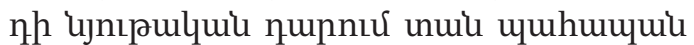
hpkzunulp tpku 5 ptipkl úpnlyuighg,

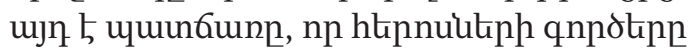

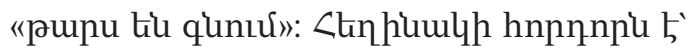

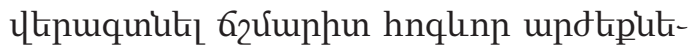

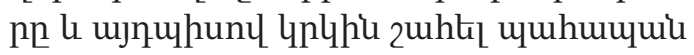

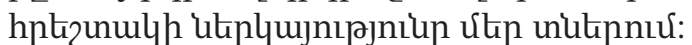

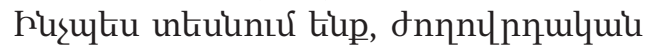

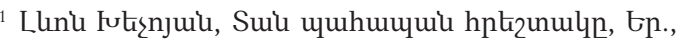
«Uluuhhun» hpuin., 2002p.: 


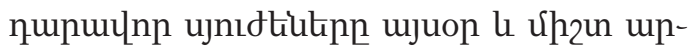
nhulquil tu husnıu jnıpupuisjnıp unp Utyumpuinn 2nıpptiphg: Uluhujun

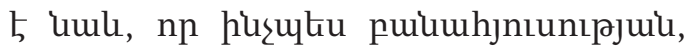

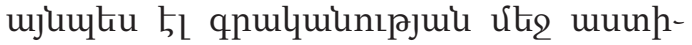

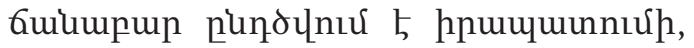
pupnju|upuinulquil ujnsdtikph qkpu-

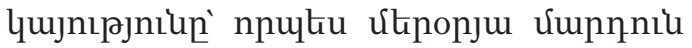
uцthl hupuquin intumul:

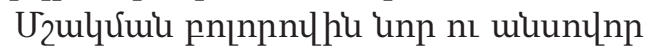
ulqpniup tiup untuinuर्u \$phqnp fupu-

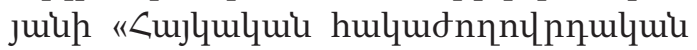
htphuplutp» (1903) qppnư: fniu hर्uuu-

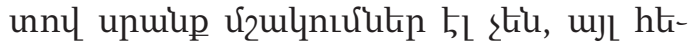

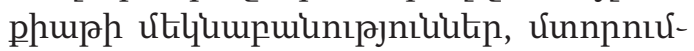

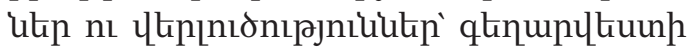

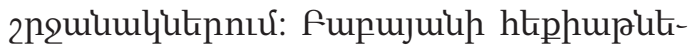
np yupdtu hupuumpu lnjudutp ihutiu:

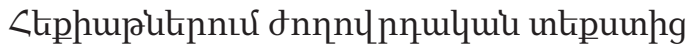
punцud nınпulh puppunujhi huin-

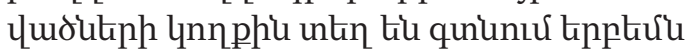

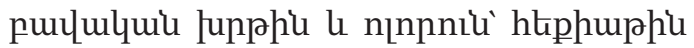
ns punpn2 n6nl ns qpulyuiu jtiquny 2u-

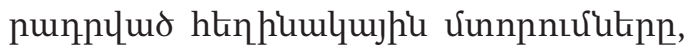

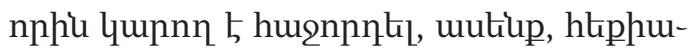
pujhi pumumulytph ulqupuqnnıрjniun

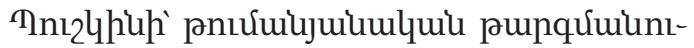

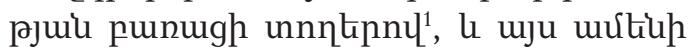

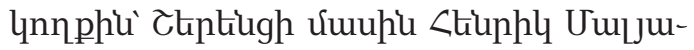

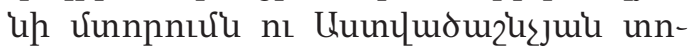
nthp hp2uunulynưn: Gnıjuuhup цnјud

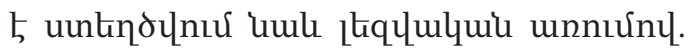
puppunh lnnphì qpulyuil pupāp n6, htionn qnupup le ujnuthu zupnituml: bl

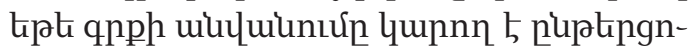

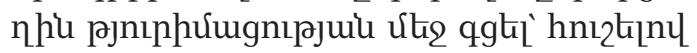

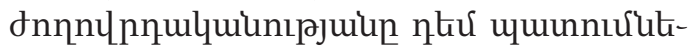

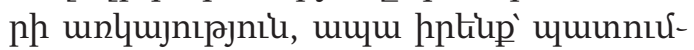
ukpp, pululyuil htnugur thitiny on-

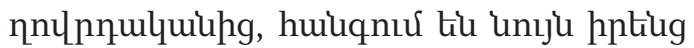

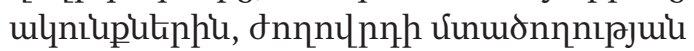
puguhujunưuin, htppuplitph hơuuun

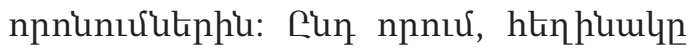

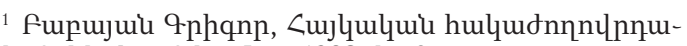
quiu htpphuplutin, En., 1903, 528 : pululumi duinp 5 htpphuplesph qhunu-

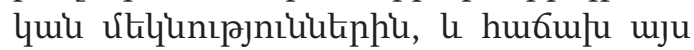
quư uju únunplh ythupknjul hpple pt

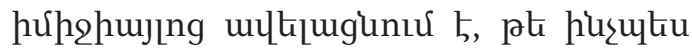

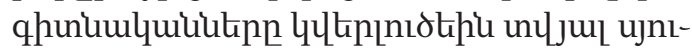

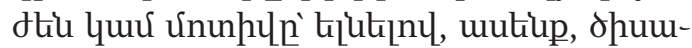
quil quu hnqkuthpnıdulyuiu untunıpjniuutuphg:

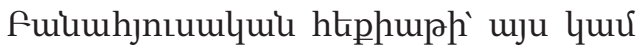
uju ulqpniupny hpuqnpdynn htinhuuqujhu úulnntúutiph pugh, ujuop qpu-

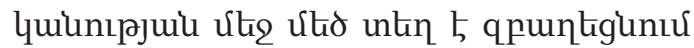

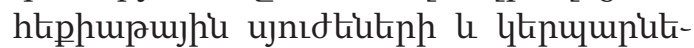
nh oqunuqnponıúp' htnhiuuluujhì hjnıuluðpnıর: ূun npnıu, uцuinulyuu ujnı-

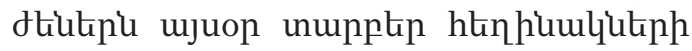

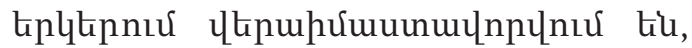

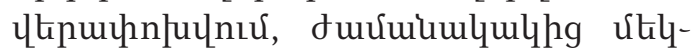
unıpjniuuten unuiunıर: Uju unkuultiunhg punpn2 ophiuul 5 durumumlyulh qpnn

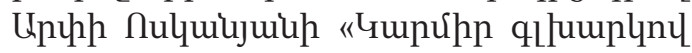

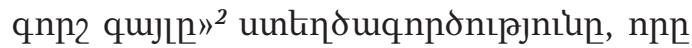
hujunuh htphupp dưumumlyulhg Uthumpuinıpjnil 5: Uluiunulyuil zuph

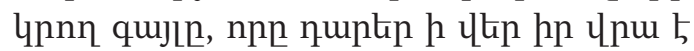

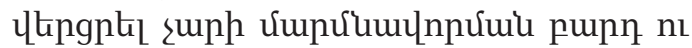
uizunphulum qnpon, uhu, hnqukl 5 hp ¿upnıpjniling, l. hkppulqui uiquú hpu-

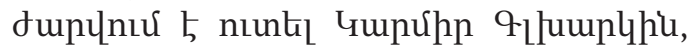
hul nizugnn qujlhi uuwuth hu hujunu-

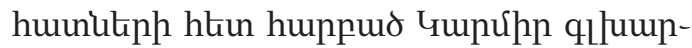

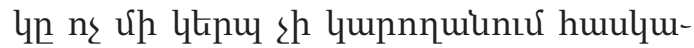

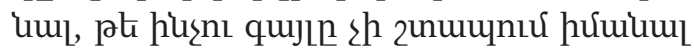
hp unuunhly unuiu untan, npukuqh htinn

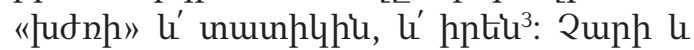

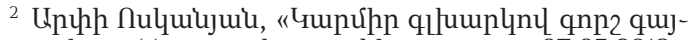
ln", http://arpi-voskanyan.blogspot.com, 07.05.2013:

${ }^{3} \mathrm{~h}$ ntuu, suphph útippu hp lnu ltingnuo l. ht-

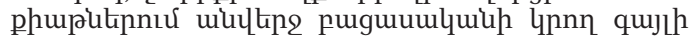

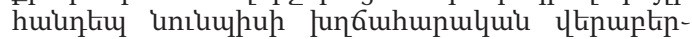
uniup ununhlutinnl th lunnılud lthotipu fou-

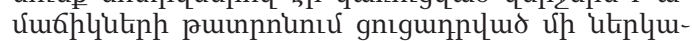
jugnıर्u, nip hpup bhu lgyud tupúp qibumph

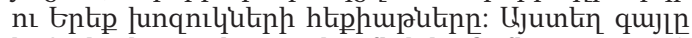

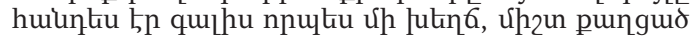

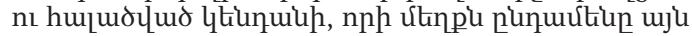
5, np husuku pnjnp ltunuuh upupudutpp, hupu

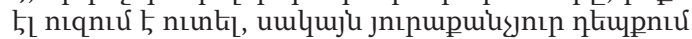




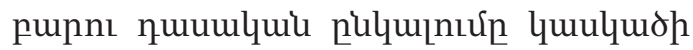

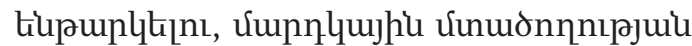
uluinqulquil htphupujhi qunuumpit-

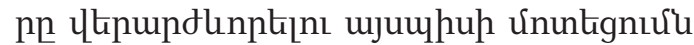
nunhuipuultu humnıl 5 upnh qpulquunıpjuin:

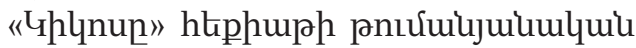

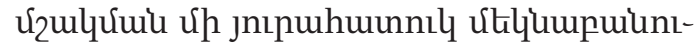
pjnilu tiup untuluntu Unर्utu Ohwijuilu «Yh-

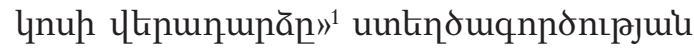

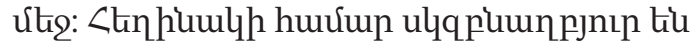
huiunhumuntu ns pt htppuph dnnnln-

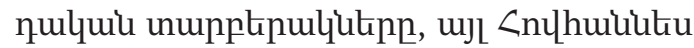

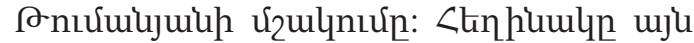
hưưqưuil 5, np «hp dnnnlpnh hłunu-

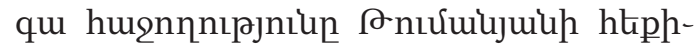

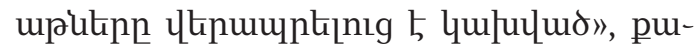

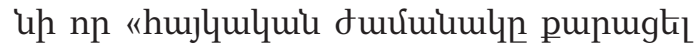
5 tipkp hqnp htppupp Utig. "2ulunpn Suinup hujh wugjuglu 5 , hujh puluinn, np sh ptiptl, "furs Guqupp" 'utplquil, hujng tpuqu ns tpuquipp, hul "Ghlyn-

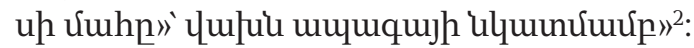

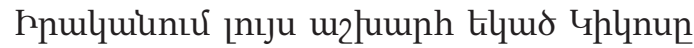

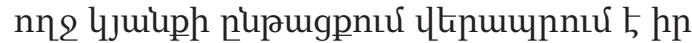
uwhp unphg nı unphg le ephnph nı dunp

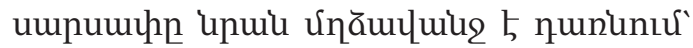

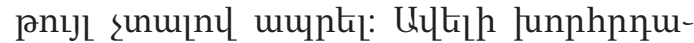

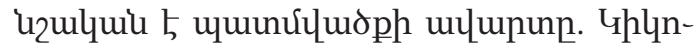

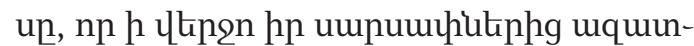

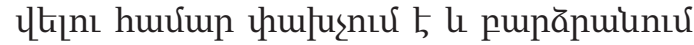

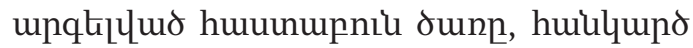

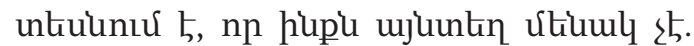

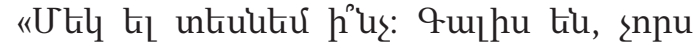

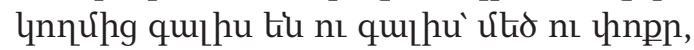
uhti-quhti no pnjnpis usphu Ghlynu tu

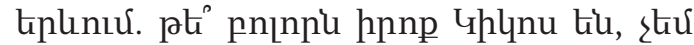
huuluuinıu: Lhpu tiu: Fnpnpp’ qnuluulnp,

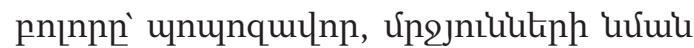

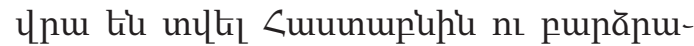
unıu tiu: Zuun-2uin tiu, Ghlnuitiph úp

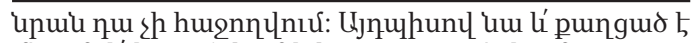

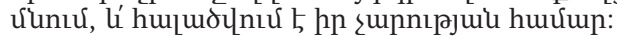

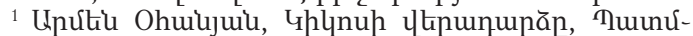

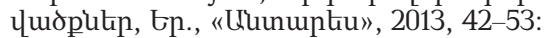

2 Gnıju untinnuर, 52 42-43:

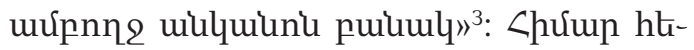

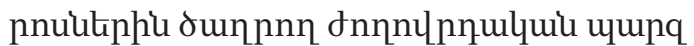

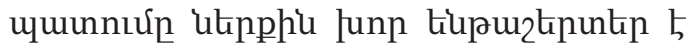

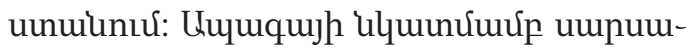

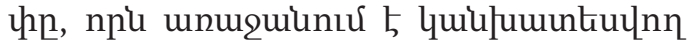

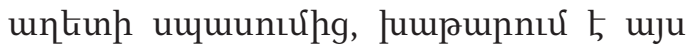

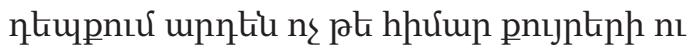

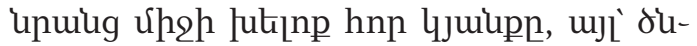
utulhp npпns:

onnnupnulyuiu htphuph unuiăhì

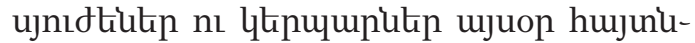

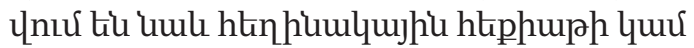

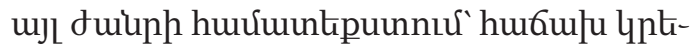

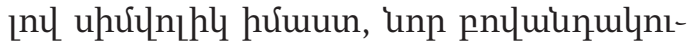

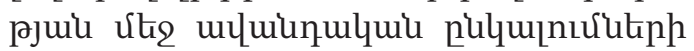

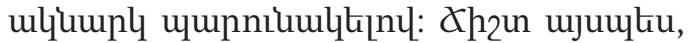
Łnpujp Unuljuili 2010p. jnıju unkuud

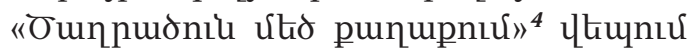

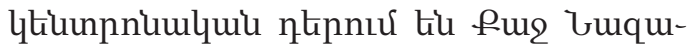
nh l. nıuunpulup lkpuquitipn: fuluun płpर्umúp puquulnp nupăud wiumpduil htipnuh ptrưu duर्umumlyulhg hpulyu-

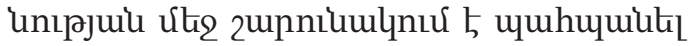

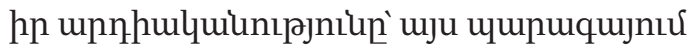

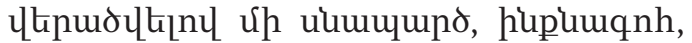
humuintils, nuduil ns upjnikuppns up-

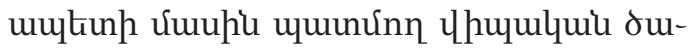

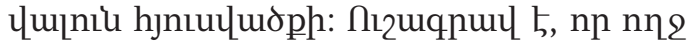

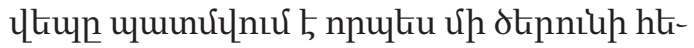
phupuuumg lnnर्uhg uwunúunn htppup, hul htinhiuuln hptiu ukpluugunıu 5 "puuwhuluup-qpunnnh» nthnus:

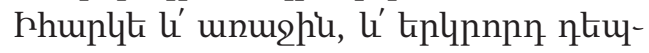
pnıu, ulyihujun 5, np qnpo nilitup n々 pts

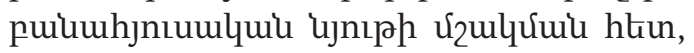
ptilnnıq le pumqnhg zuin htinugurd, ujl puiuuhjnıunıрjuil qpulquiuugumo unuppkpulukph htinuqu qupqugntúut-

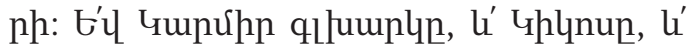
fus Guqunp, h ulqpulut duniun unit-

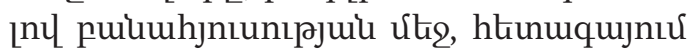

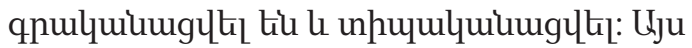

\footnotetext{
3 Gnıju unknnıu, 52 51-52:

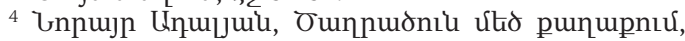

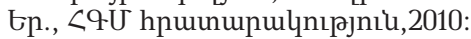




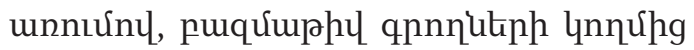
uju utipuniudotuph oqunuqnpontứu upntilu hłnns 5 puiumhonıumlyuil htphuph

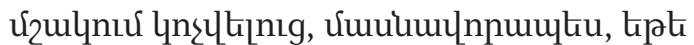
hw2lh unitiup, np fur turqup lnsunn

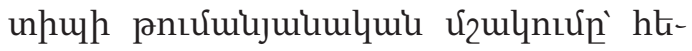

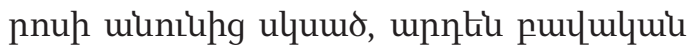

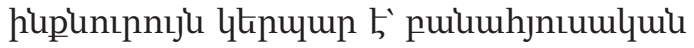
unupptepulhg htenugud:

onnnцpпuluiu htphupitpp qnulyuil

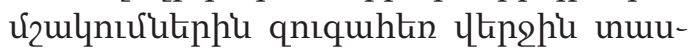

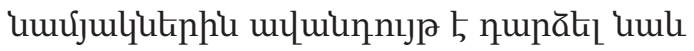
puivuqken-puiumhuluptikph lnnúpg

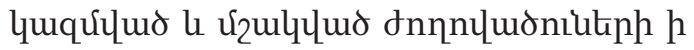

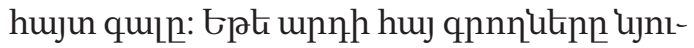

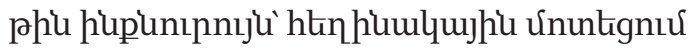

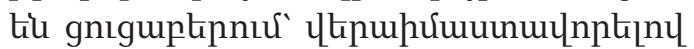

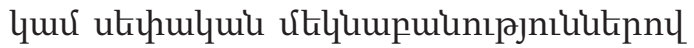
ukplujughtinnl uju, uuw htphuphtph pumqnkphi hupuquin, umlquju jtqum-

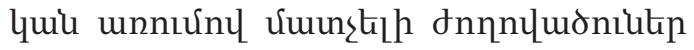

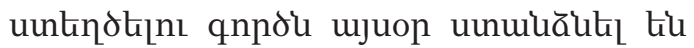

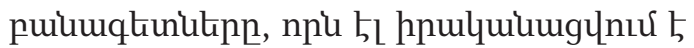

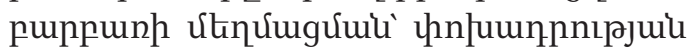
Guiuuuuphny:

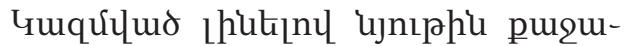

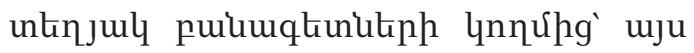

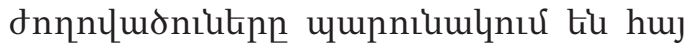

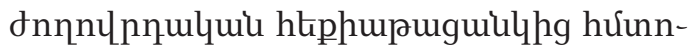
ptiu puunplud, luinnsghl no qunuum-

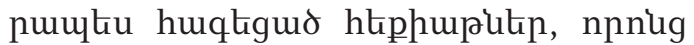

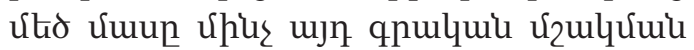

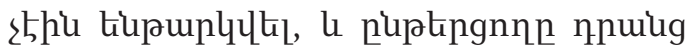
quinnn 5p duinp lhikl uhuju puppunujhi hpuunupulnıpjnitukph uhengnu:

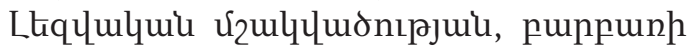

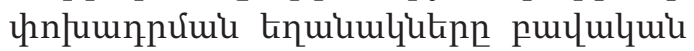
unupptep tu: Ept ú dnnnцuonınıর puppunp hiupulnphiu umhumilud $5, \mathrm{l}$

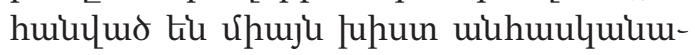
lh ăliknp, uuwu ujnıuniu jtiqnil qptept

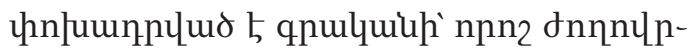
nuluuiu puntip ns upunuhujunnıpjnikitep uhuju umhuquit]nu: 9npдp unugurumpunly huj hpulumunıpjuiu Ute Ununu2tu Guqhujuiu $5 p$, npp, thutinl «<uj dnnnlpnulyuil ht-

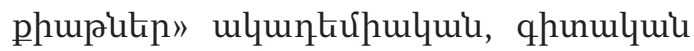
hpuunupulnıрjuil unueghu huinnpitiph

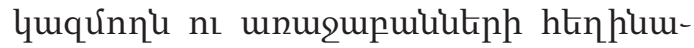

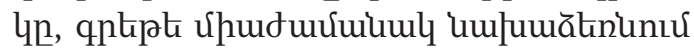
5 'uul dnnnlpnulyuil htphuplitph qt-

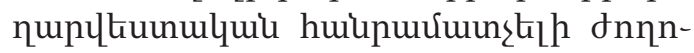

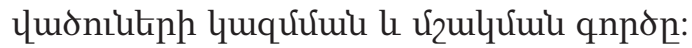
1956p. jnıju 5 untuintu upu úulquo ht-

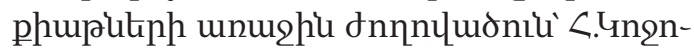
juiup ulqupuqupnnứutipnl, npp lthpuh-

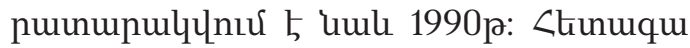

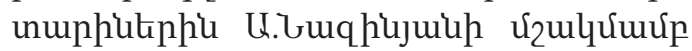
Łnuju tiu untuunıu dnnnцpnuluiu htpph-

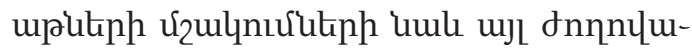
oniutp' 1960p., 1967p., 1986p. l 1995p: உ'un

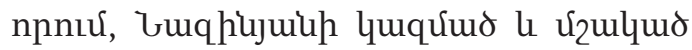
hłphuplitiph uju dnпnцuonitukn jnıju

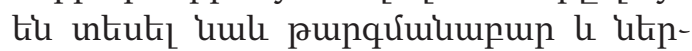
qujugltil ununuumhर्umujuil puptingnnhì nnıutptiu (Unuluu, 1969), lhunцtuptiu (Uhjujnıu 1979), juunh2kptiu (ก-hqu 1981) l

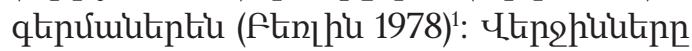

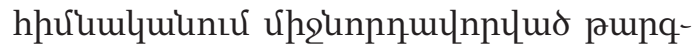

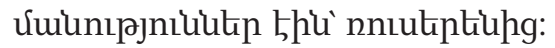

Ujuuhunl, npi $5 p$ Guqhijuilh v2ul-

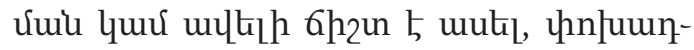

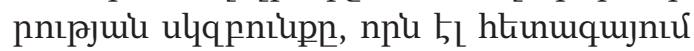

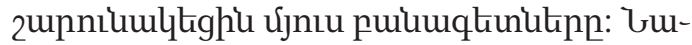

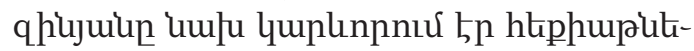

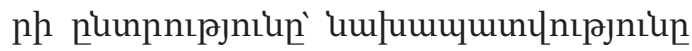

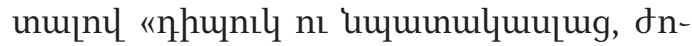

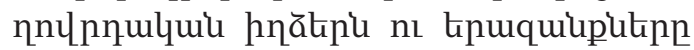

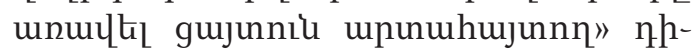

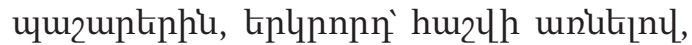

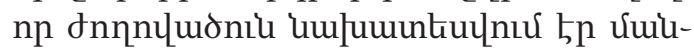

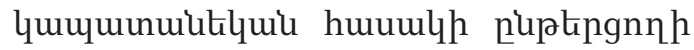

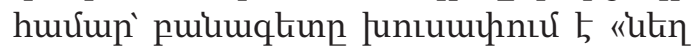
puppunujhi la ounup puntephg nı upunu-

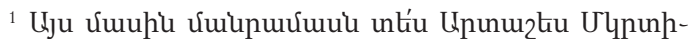

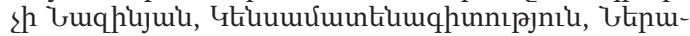

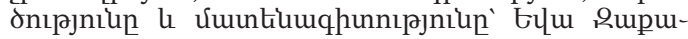
pjuluh, Gpluwu, «Unın'up» hpuun., 2004:
} 


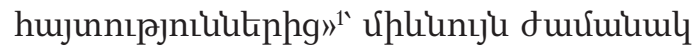

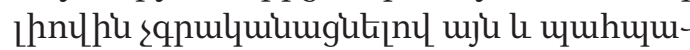

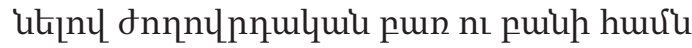

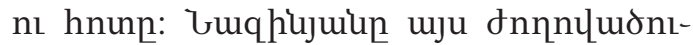

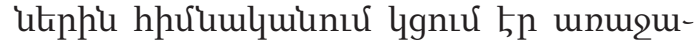
puiluten, nıp ưunstilh puguinnnıpjnitu

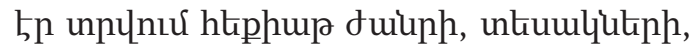

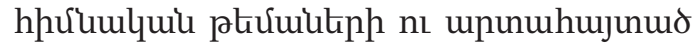
qunuumuputiph umuph: $\mathrm{h}$ lthen, npuku

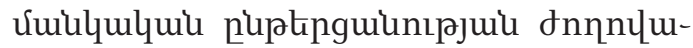

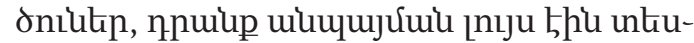
untu qtintighl ulqupuqupnnứutinnl, ú puil, nnp plumlumumpup pugulqujnı $5 n$ dnnnцpnulquil htphwpitiph qhunulumi hpuunupulnıpjnıkutennı์: Guqhujuup uju dnnnluoniktenp puluquil uto

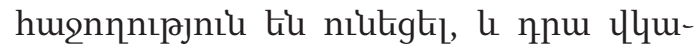
jnıpjniuu tiu npuiug lthpuhpumupulnn-

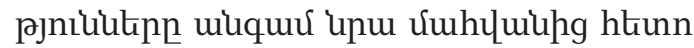
lu úpųl ưtin optinn²:

onnnunnulumu htiphuputiph uxumum-

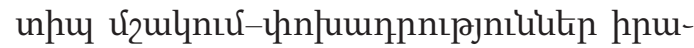
quiumgnti 5 umb puiumqtion bhilh $\mathrm{Cu}$ lnnjuiun: 1977p. jnıu 5 untulunıu upu l

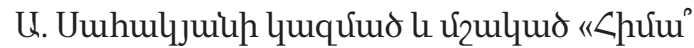
kiu nıqnuर्u, pte htiunn» qpulquu-qtenup-

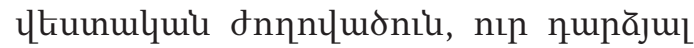

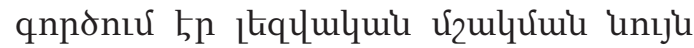

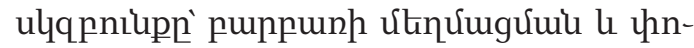

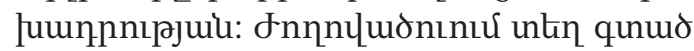

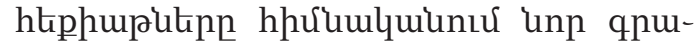

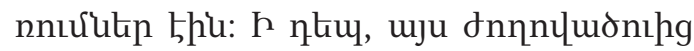
Łplnı unuph uiug'1980p., nnıju 5 unkuunıu 孔. Lulnnjuluh quqúur unptil qhunu-

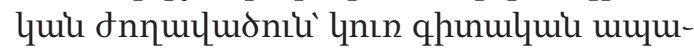
puinnl le htpphupltiph' wuphti-pnưh-

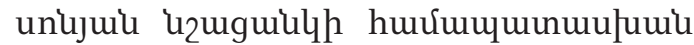
nuuulquinqniúnl, nıp unten tiu quiuntu

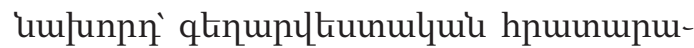

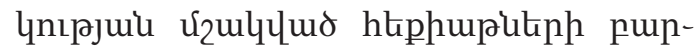

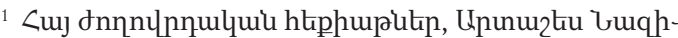

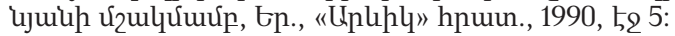

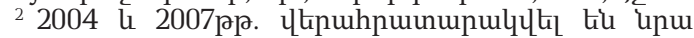

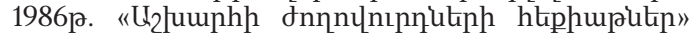

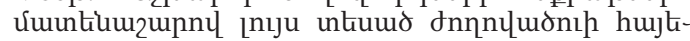
phit lu nnıutiptiu numptipulutipn:
}

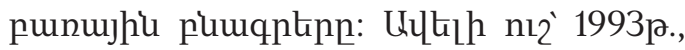
unumqnunuर 5 Gtilh <ulnnjuil quq-

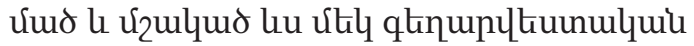
১nпnumoni” «3nр lupuinulquiu htppup»

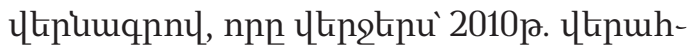
purnunulyttl b "Unteq" hpumunuly¿nıрjuiu lnnứhg: ¿'un nnnư, l' 1977p., l' 1993p. dnnnцuoniutenn juर्umuqnumo thu

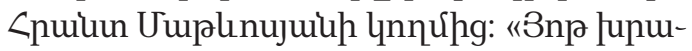
unuluu htphup»" dnnnulurnınıu untn

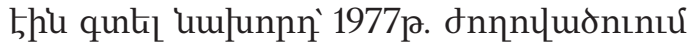

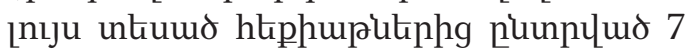

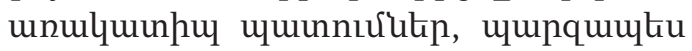

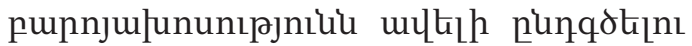

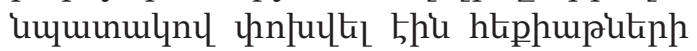
ytupumqntinn' hnjumphiutiny huenn liknund numplud uumgluoputinnl: Ujuuku. «nl hí wuh, hpliu quiup» (52

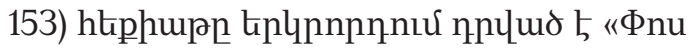

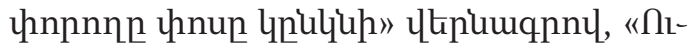

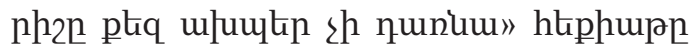

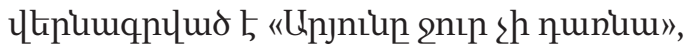

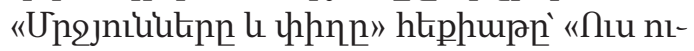

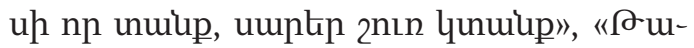

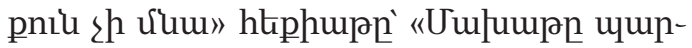

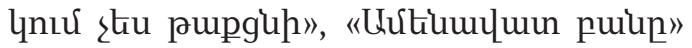

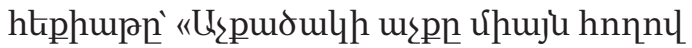

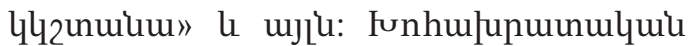
htppuplitiph uju unpphl hnilig l' pn-

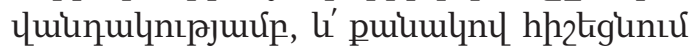

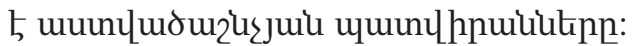

fuiumqtinutiph lnnúng quqưumo ht-

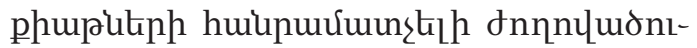

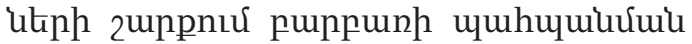
untiumlitinhg unumăbumuniu 5 Utinqtis

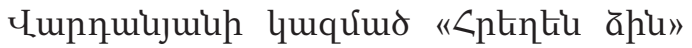
(1981) qhnpn, npu uर्uफnџnıu b hŁphupukn, unulitin, knqtin, unud-uuug-

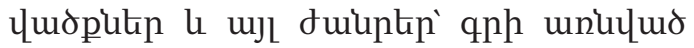

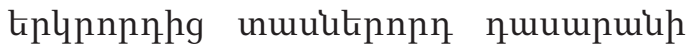

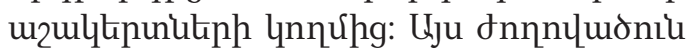
hus-np unnounl unujuhul sh bl qupt-

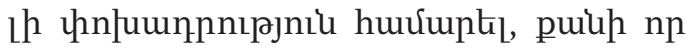

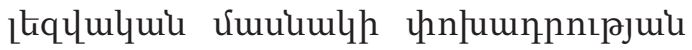

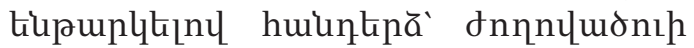




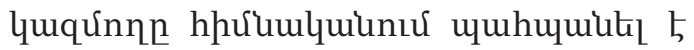

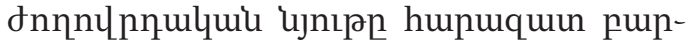
punnl: Puilh np onnnluonil kuruw-

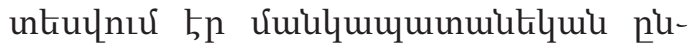

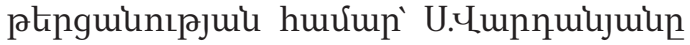
jnıpupuisjnıp htphurp tlipgnư unulhu

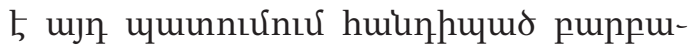
nujhi wihwulumum puntiph thpphl guillkn: Lhnulhu qpulquiu ghquh tiu hn-

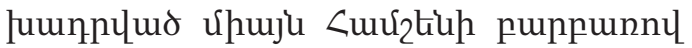
ưunnıরitinn: টnıju ulqpniuputinny 5

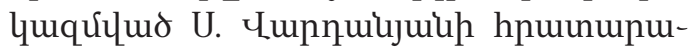

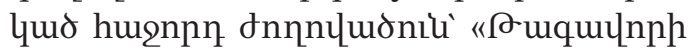
tinuqutinp» (2003p.):

fuppunh huintiu ưumu hnquons

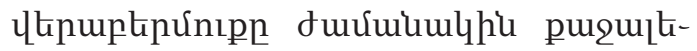

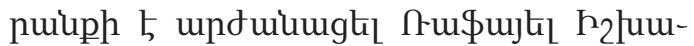

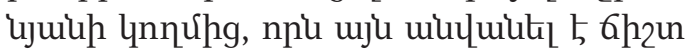

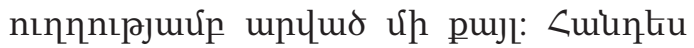

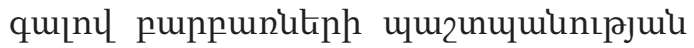

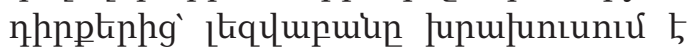

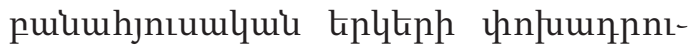

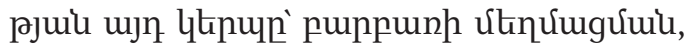

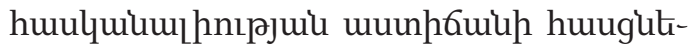

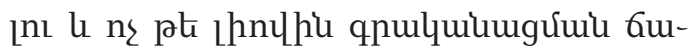

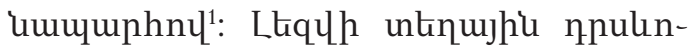

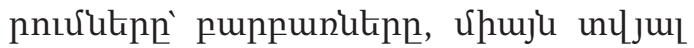

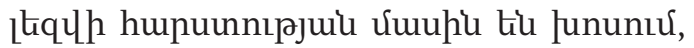
npnup unuguplynư tiu ăluwjhu hupnıuun puqưuquinıpjniu: Uuznı2u jtiqumumu

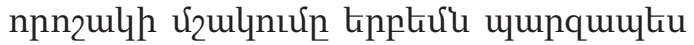

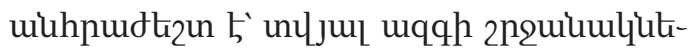

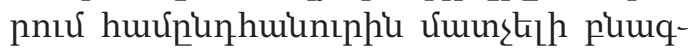

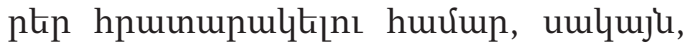
qunonsu tiup, np guilumeh \&5 ujnuhup

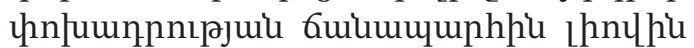
qpulquiumgitil puiumhnsumlquiu pumqpkipn, $25^{\circ}$ np puiuwhjnıunıpjniun qnjnıpjnilu nilup htilg ujn puppunitiph úp2ngnu: Guil zuin qtintighl puppunujhi ălikp nı upunuhujunnıpjnikukn, unuikg

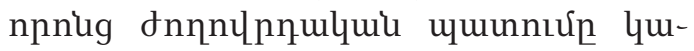

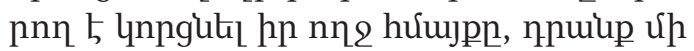

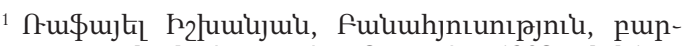
pun, upnhulquinıpjnıน, "9upnıł», 1983, рһц 1:

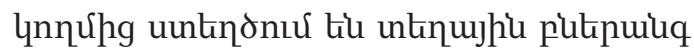

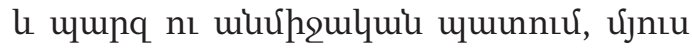

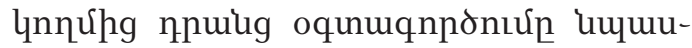
unnư 5 punpunujhí ujnuhup npuln-

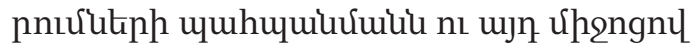

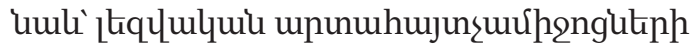
hupuunugưuin, jtqqlh unupptip 2 tipunteph ujLnunnuipujhi unupptinulytiph úto ưuhnıuunh ăluuцnnưuiun:

fuiuwhynumuluiu htiphupitiph qta-

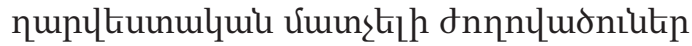

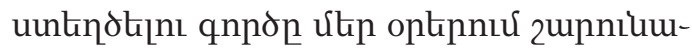

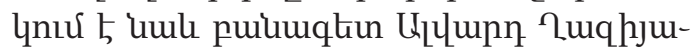
un: 2007p. [nuju b untuinnu U. 2.uqhjuiuh

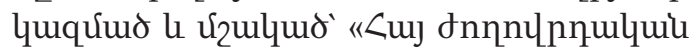

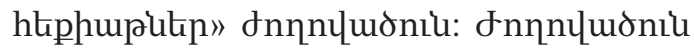
hiphumunpu 5 humlyuuku uju pugnn $\mathrm{l}$

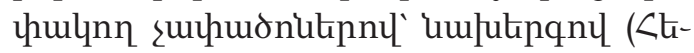

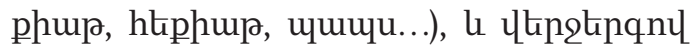
(Uuqpny tiluiu unılu no quunniu...): fn-

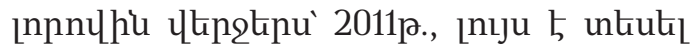

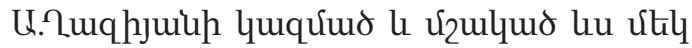

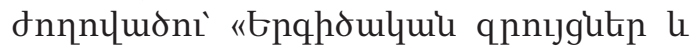
qump6urununıpontuitip" ytipumqnnl: 2urhukpnl pululumi unpn' qptipt qn-

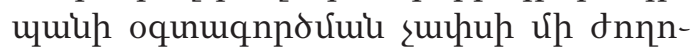

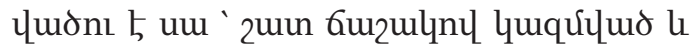

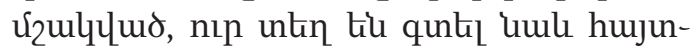
uh tinqhoulqui htphuplitph hưưnnu unupptipulutip: Ujuuphup dnnnцuðnilutne guiuluul til onnnunnulquil ujnıp

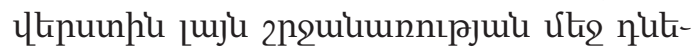
ఏnt unnứnu:

Ujuuhunl, ulihumju b, np ptil dn-

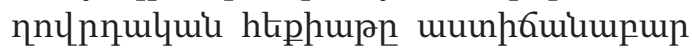
lnngunuर 5 hp uluminulyuil ltikgu-

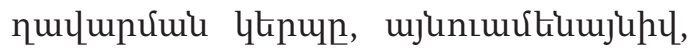

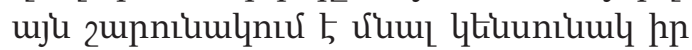
qnunцuo unupptipulynl: nt ptil uju ltip-

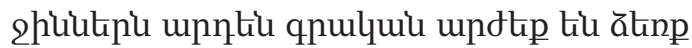
pŁnnıर्u, uulumu র্ pnıрjuiu ulqpnilputiphg, umluuju 2w-

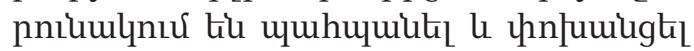

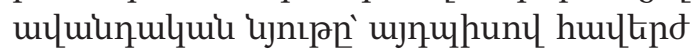

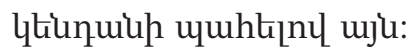




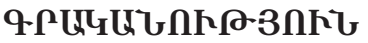

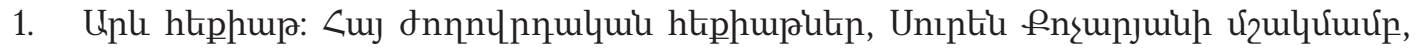
bp., 1979:

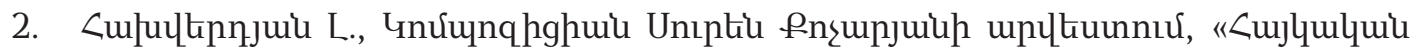

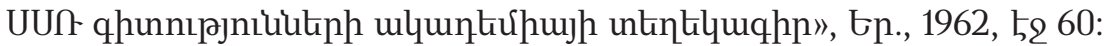

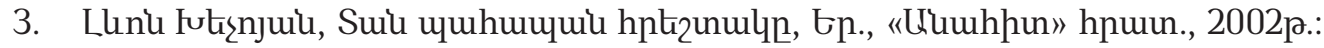

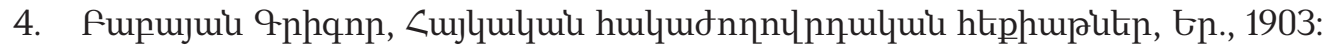

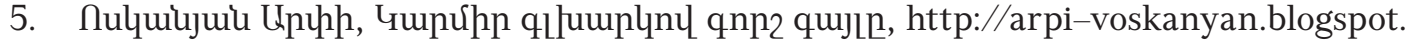
com 07.05.2013:

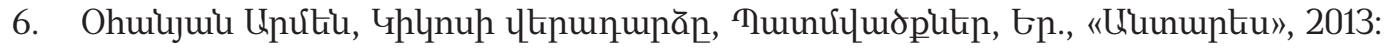

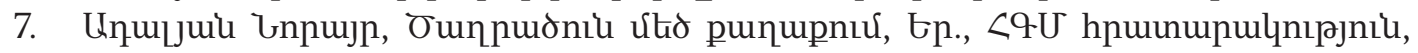
2010:

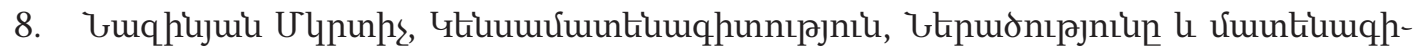

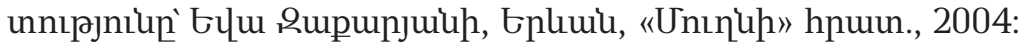

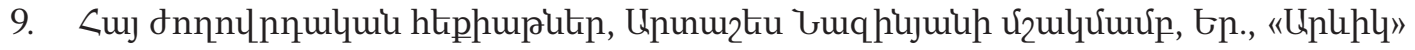
hpuun., 1990:

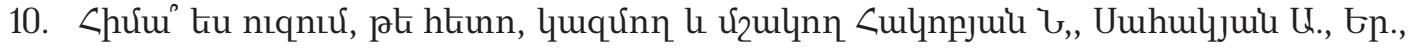
1977 р.:

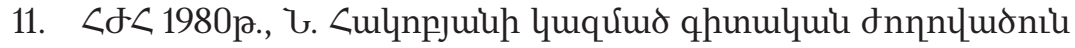

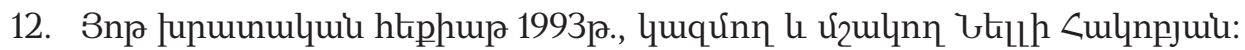

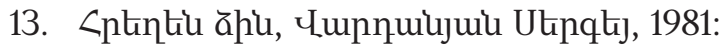

14. r 1983, phu 1:

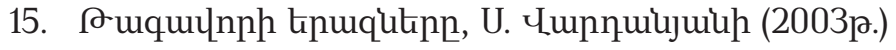

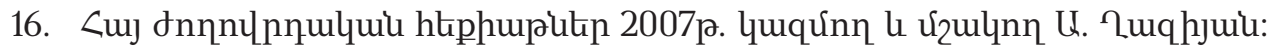

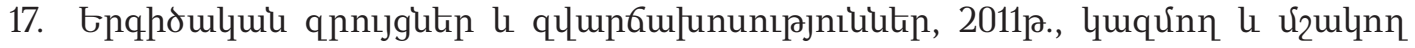
U. Ruqhjuu:

\section{СОВРЕМЕННЫЕ ТЕНДЕНЦИИ ОБРАБОТКИ И ПЕРЕЛОЖЕНИЯ ФОЛЬКЛОРНОЙ СКАЗКИ}

\section{НВАРД ВАРДАНЯН}

\author{
Преподаватель факультета армянской филологии Ереванского государственного \\ университета, кандидат фил. наук
}

\section{НАРИНЕ ВАРДАНЯН}

Заведуюший отделом обеспечения качества образования Международного научнообразователного иентра Национальной Академии наук РА

Несмотря на то, что в наши дни фольклорная сказка не столь популярна, как раньше, в литературе она продолжает развиваться. Авторские подходы к народному материалу сегодня достаточно различны, начиная с литературных обработок, заканчивая языковыми переложениями. 
За последние десятилетия сборники народных сказок, составленные на основе литературной обработки, хотя и мало, но издавались. В них содержатся близкие к оригиналу ценные обработки, как обогащенные своеобразным авторским подходом (А. Кочарян, Л. Хечоян), так и созданные по совершенно новым необычным принципам (Г. Бабаян). А некоторые писатели представляют известные сказочные сюжеты, переосмысливая их или наделяя современными комментариями (А. Восканян, А. Оганян, Н. Адалян).

Помимо художественных обработок в настоящее время на литературном рынке большое место занимают сборники, составленные фольклористами, в которых народный материал подвергнут языковому переложению, а непонятные и замысловатые диалектные формы представлены в смягченном виде (А. Назинян, Н. Акопян, А. Газиян, С. Варданян). Такие популярные сборники весьма способствуют распространению народного материала на новом уровне.

\title{
CONTEMPORARY TRENDS OF DEVELOPMENT AND EXPOSITION OF FOLKLORE TALE
}

\author{
NVARD VARDANYAN \\ Lecturer of the Department of Armenian Philology, Yerevan State \\ University, PhD in Philological Sciences
}

\section{NARINE VARDANYAN \\ Head of the Quality Assurace Department, International Scientific-Educational Center of National Academy of Sciences of Republic Armenia}

Though nowadays the folklore tale is not told to that extent it used to, it continues its development in literature. Author approaches to folklore material is currently quite different starting from literary adaptation to language expositions.

Only a few collections of folklore tales created through literary adaptations have been recorded in recent decades. They contain both high-quality adaptations close to material with unique author approaches (S. Qocharyan, L. Khechoyan) and completely new adaptations created with extraordinary principles (G. Babayan).

Some authors give new meaning or contemporary interpretations to the plots of famous folklore tales (A. Voskanyan, A. Ohanyan, N. Adalyan).

Besides literary adaptations, the collections drawn up by folklorists take up a large place in the contemporary literary market, where folklore material is subjected to language expositions, while non-understandable and abstruse dialectal forms are mitigated (A. Nazinyan, N. Hakobyan, A. Ghaziyan, S. Vardanyan). Such popular collections are very favorable to put folklore material into circulation once again. 